\title{
DIGITAL TECHNOLOGY Introduction of an electronic weekend handover system at a London university teaching hospital
}

\author{
Authors: Stephen $]_{\text {Mounsey, }}{ }^{A}$ Halima Amer ${ }^{B}$ and Michael Yeung ${ }^{C}$
}

Handover has long been identified as a potential source for error that can have direct consequences for patient care. The current weekend handover system at University College Hospital, London, involved a verbal handover meeting with a variety of methods used to collate information. A new handover system was introduced that involved the pre-population of a spreadsheet before attending the handover meeting. Doctors involved in the handover process were canvassed for opinion before and after the introduction of the new system, and logistical data were collected. The new system reduced the amount of time spent preparing for, and delivering, handover, and was deemed to be safer and to improve adherence to the Royal College of Physicians' guidelines for handover, including specification of level of doctor to perform handover and prioritisation of handover.

\section{Introduction}

Effective, safe and comprehensive handover is an essential aspect of providing patient care. International research has identified poor handover as a major contributing factor to potential harm. Handover guidance has been issued by the Royal College of Physicians (RCP) and the Royal College of Surgeons (RCS), and by the UK General Medical Council in an attempt to reduce risk. ${ }^{1-6}$

The change to doctors' working hours with the adaptation of 7-day working has resulted in more frequent handover episodes between medical colleagues. The weekend handover is often the most complex - frequently with complete changeover between medical staff caring for patients. This results in significant potential for human error and, as a consequence, negative impacts on patients. ${ }^{7}$

Although overarching principles of handover are the same nationwide, each hospital varies in size, specialties, team members and resources. As a result, handover delivery continues to be variable. $^{7-13}$ University College Hospital, London, is a large, quaternary-referral teaching hospital with over 650 inpatient beds at its main site. Specialties include a respiratory medicine team, three medicine for the elderly teams, four gastrointestinal teams

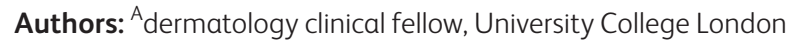
Hospital, London, UK; ${ }^{\text {B }}$ specialist registrar, Clinical Pharmacology and Therapeutics, University College London Hospital, London, UK; Consultant physician, University College London Hospital, London, UK and a clinical pharmacology team. The weekend medical cover was provided on a rota basis with two first-year postgraduates (FY1s), a second-, third- or fourth-year postgraduate (FY2/ CMT) and a fifth-year postgraduate or higher (ST3+) from these specialties (Fig 1). Handover for the weekend system comprised a Friday afternoon meeting, where outgoing junior doctors provided paper ward lists for all of their patients and outlined those who would require intervention over the weekend. Tasks to be completed over the weekend were then consolidated onto a spreadsheet by the incoming team, paper copies of which were distributed (Fig 2).

The system required improvement. The nature of the meeting was unstructured, protracted and time inefficient. Preliminary research showed that the time spent attending handover meetings was often inappropriately long and unproductive, with teams queuing to hand over and often staying past the end of their shifts. The lack of a structured handover form provided great interdoctor variability in terms of the data recorded. Further preliminary data showed that this was often noncompliant with the guidelines suggested by the RCP (Table 1).

Once the consolidated handover had been produced and distributed, there was no way to update the spreadsheet, preventing team members from accessing a 'live' version to add new tasks and to mark others as completed, or to feedback to the weekday team returning after the weekend.

\section{Aims}

We developed an electronic handover pro forma that could be accessed by multiple doctors simultaneously, allowing standardised pre-population before attending the handover meeting. We wanted to have $100 \%$ compliance not only with the new electronic system from all outgoing teams to standardise the handover process, but also with the guidelines set out by the RCP. Using the new system, we aimed to reduce the time spent both preparing and delivering handover (Box 1).

\section{Methodology}

Questionnaires were issued to all 23 doctors working on the general medicine weekend ward cover rota. Data collected included opinion on importance of handover, current method of handover, time spent preparing handover, time delivering handover, number of patients handed over, prioritisation of tasks, identification of level of doctor to perform task, and opinions on the safety of the handover system. 


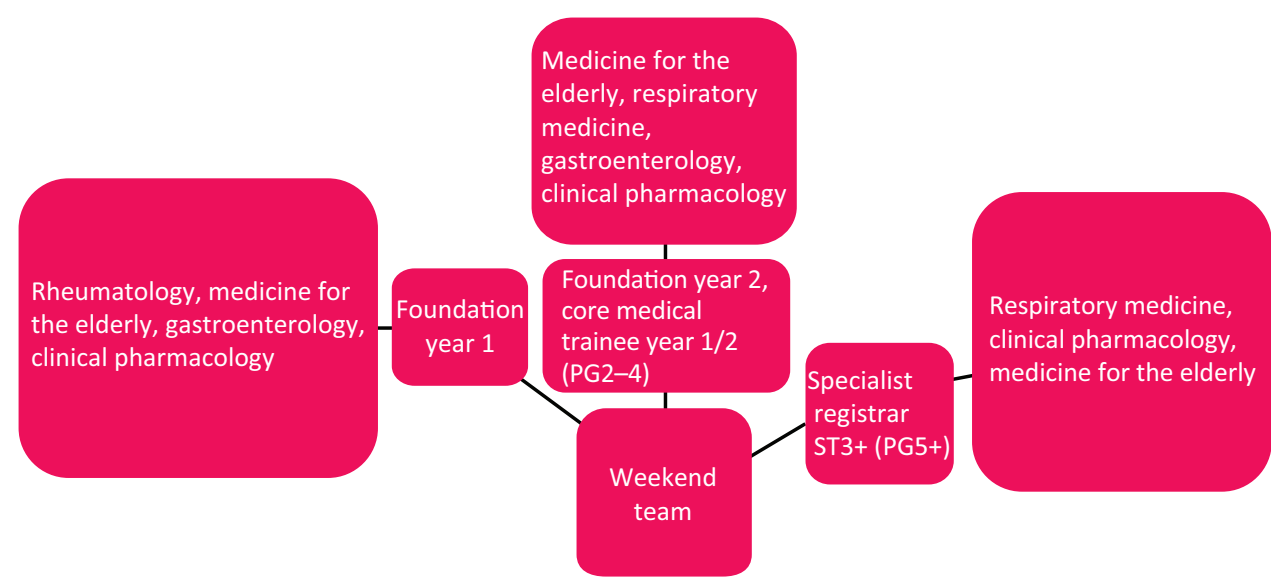

Fig 1. Overview of weekend team make-up.

The electronic system was created by a cohort of doctors, which included a medical consultant, medical registrars, core medical trainees and foundation doctors. The system was created using Microsoft Excel and was saved on a shared drive on the hospital network, to which only named doctors had access. The file was password protected. A new file was created from an existing protected template each week.

After data collection, the new electronic system was introduced. Three months after the initial introduction, the same cohort of doctors then recompleted the same questionnaire.

After the initial introduction of handover, we optimised the electronic handover template over a 2-month period. Changes orientated around making mandatory data entry fields and ensured that all necessary information was correctly recorded. Several technical issues with regards to the joint sharing and saving of the spreadsheet were identified and addressed.

A 'how to' guide was produced for doctors who were new to the system. This orientated particularly around the use of Microsoft Excel, how to create a new handover file and how to complete it. We also provided advice on filtering the form and printing.

\section{Results}

After the introduction of the new electronic system, doctors completed the same questionnaire as previously. Twenty-three questionnaires were returned, representing all the foundation doctors and core medical trainees during one medical rotation. The results are outlined below.

\section{Compliance with Royal College of Physicians' guidelines}

The new handover system had 100\% compliance with the guidelines issued by the RCP.

\section{Importance of handover}

After the introduction of the new handover system, the importance of the handover remained relatively unchanged. Most doctors felt that handover was very important $(56.5 \%$ and $52 \%$, foundation doctors and core medical trainees, respectively) or important ( $39.1 \%$ and $47 \% \%$, foundation doctors and core medical trainees, respectively). Before introduction, $4 \%$ of doctors felt handover was somewhat important.

\section{Method of handover}

All doctors completed the electronic spreadsheet and attending the handover. Before the introduction of the new system, a variety of methods had been used, including formal handover (18.9\%), verbal handover $(45.9 \%)$, handwritten handover $(21.2 \%)$ or electronic handover (21.6\%).

\section{Time preparing for handover}

The introduction of the electronic handover reduced the amount of time spend preparing for handover. Before the introduction of the new system, the mean time spent preparing was $24 \mathrm{~min}$. After the introduction of the new system, this had reduced to $18 \mathrm{~min}$; a reduction of $\sim 6 \mathrm{~min}$ or $25 \%$.

\section{Time spent in handover}

A similar reduction in time was seen with time spent in handover. Before the introduction of the electronic system, the mean time spent handing over was 22.5 min, compared with 12.4 min after the introduction. This resulted in a reduction of $\sim 11$ minutes or $45 \%$.

\section{Percentage of patients handed over}

The introduction of the new system also saw a statistically significant reduction in the number of patients handed over. Before the introduction of the new system, a mean of 58.7 patients were handed over, which reduced to 46.5 after the new system was introduced; a reduction of 12.2 patients or $21 \%$.

\section{Prioritisation of handover}

Before the introduction of the new handover system, myriad techniques were used to highlight the importance of jobs, with $17.4 \%$ never identifying importance, $43.5 \%$ of patients verbally handed over, $13 \%$ writing the importance, and $30.4 \%$ both writing and verbally communicating importance. This changed to $0 \%$, $0 \%, 4.3 \%$ and $95.7 \%$, respectively.

\section{Identifying roles for handover}

Identification of the doctor required to perform the job in the handover was present in $82 \%$ of patients before the introduction 


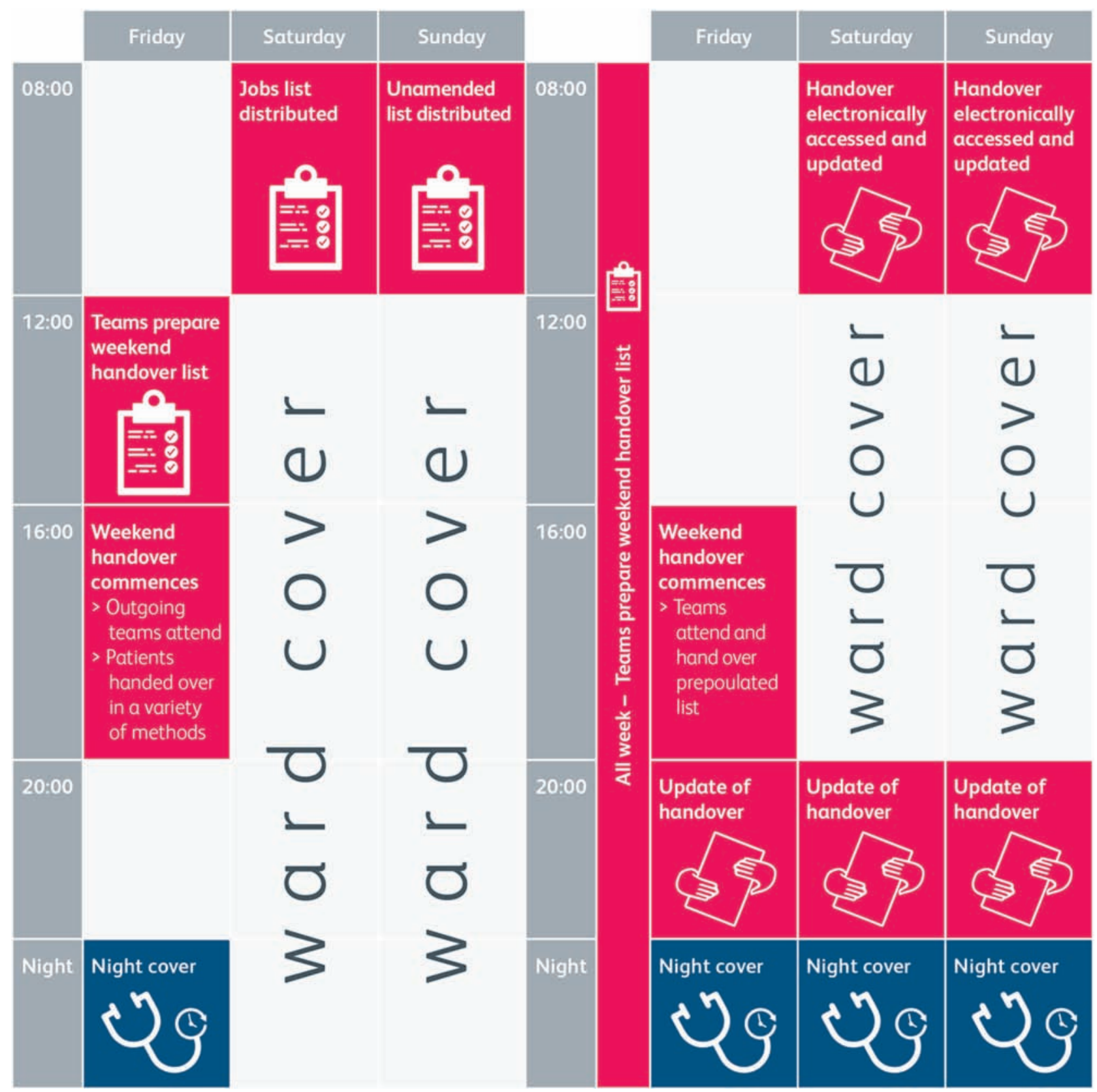

Fig 2. Process map of handover before and after introduction of new system. ${ }^{a}$ This change in process results in handover taking less time complete.

of the new handover, and increased to $91 \%$ of patients after the introduction.

\section{Safety}

Before the introduction of the new system, $65.2 \%$ of doctors thought that the handover process was safe, which increased to $100 \%$ after the introduction.

\section{Discussion and limitations}

The methods, practices and culture of handover varies widely throughout both the UK and internationally, with a variety of different methods used, including verbal, ${ }^{8}$ written ${ }^{9,10}$ and electronic systems ${ }^{7,11,14-16}$. After appraising the literature for the types of system previously introduced, we adopted a new electronic system. This aimed to address the unstructured nature of weekend handover and effectively produced a time-effective solution, which should translate into improved efficiency, as shown by the reduction in time spent creating and delivering handover. We hope that this will have future ramifications for medical staff, and help to alter the culture within teams with regards to handover.

\section{Lessons learned}

The introduction of a new electronic system allowed more complete and accurate data recording for handover, which 
Table 1. Comparison of compliance with Royal

College of Physicians' guidelines before and after introduction of new handover system

$\begin{array}{lll}\text { Patient information } & \text { Pre } & \text { Post } \\ \text { Patient name, date of birth and } & \checkmark & \\ \text { hospital number } \\ \text { Patient location }\end{array}$

was easily accessible from a range of sites around the hospital simultaneously, resulting in comprehensive data being available quickly. The introduction of the new system was received positively by the current cohort of doctors and improved their overall sense of safety with regards to handover. By highlighting handover as an issue, we were also able to educate doctors about what issues needed handing over; we felt that this helped reduce the total number of jobs handed over.

The reduction in time of handover also helped junior doctors comply with the European Working Time Directive by allowing them to finish their shifts in a timely fashion.

Future aims for the project include integration of the system into junior doctor induction, ongoing assessment of correct usage of the system, along with adaptations as needed.

\section{Limitations}

Future work with regards to the development of handover orientate around education and technological. With regards to education, the importance of handover and how to practically deliver a handover needs to be instilled in all doctors from a junior level upwards. This study highlighted fundamental flaws within the process and reasoning behind handover by junior doctors, and we aim to address this in future studies. Ideally, the system would
Box 1. SMART aims for handover development

$\mathrm{S}>$ Produce a new system for weekend handover

$>$ Be accessible by multiple users from multiple areas

> Adopt RCP guidelines and standardise handover

> Assess impact upon handover processes

$\mathrm{M}>$ User testing to measure accessibility

> Questionnaire to doctors involved in handover to assess impact

> Comparison of final system to RCP guidelines

A > Involvement of all subspecialties of medical directorate

$>$ Creation of secure, shared drive to save and access handover

$\mathrm{R}>$ Changing work patterns of doctors means more frequent handover

> Previous handover had been shown it needed improvement

$\mathrm{T}>$ Initial pilot must be completed during 4-month rotation to allow for assessment of impact upon same cohort

also undergo further changes to allow an even more user-friendly interface, with 'pop-up' box entries to overcome technological issues.

\section{References}

1 Roughton V], Severs MP. The junior doctor handover: current practices and future expectations. J R Coll Physicians Lond 1996:30:213-4.

2 General Medical Council. Good medical practice. London: GMC, 2013.

3 Royal College of Physicians. Acute care toolkit 1: handover. London: RCP, 2015.

4 Royal College of Physicians. A scoping project: handover - the need and the practice. London: RCP, 2010.

5 Junior Doctors Committee. Safe handover: safe patients. Guidance on clinical handover for clinicians and managers. London: BMA, 2012.

6 Royal College of Surgeons. Safe handover: guidance from the Working Time Directive working party. London: RCS, 2007.

7 Govier M, Medcalf P. Living for the weekend: electronic documentation improves patient handover. Clin Med 2012;12:124-7.

8 Raduma-Tomas MA, Flin R, Yule S, Williams D. Doctors' handovers in hospitals: a literature review. BMJ Qual Saf 2011;20:128-33.

9 Michael E, Patel C. Improving medical handover at the weekend: a quality improvement project. BMJ Qual Improv Rep 2015;4:u207153.w2899

10 Bethune R, Campbell K, Rose A et al. Improving weekend handover between junior doctors on medical and surgical wards. BMJ Qual Improv Rep 2014;2:u483.w1045.

11 Curtis O, Fisher R. Improving medical SHO weekend handover at a tertiary referral centre. BMJ Qual Improv Rep 2013;2:u697.w971.

12 Saifuddin A, Magee L, Barrett R. 'Chase CRP', 'Review patient': Improving the quality of weekend medical handover at a London teaching hospital. BMJ Qual Improv Rep 2015;4:u201656.w1919.

13 Ferran NA, Metcalfe AJ, O'Doherty D. Standardised proformas improve patient handover: audit of trauma handover practice. Patient Saf Surg 2008;2:24.

14 Vithlani R. Introduction of a new electronic medical weekend handover at Tunbridge Wells Hospital. BMJ Qual Improv Rep 2015;4:u204755.w2342. 
15 Raptis DA, Fernandes C, Chua W, Boulos PB. Electronic software significantly improves quality of handover in a London teaching hospital. Health Informatics J 2009;15:191-8.

16 Cheah LP, Amott DH, Pollard J, Watters DA. Electronic medical handover: towards safer medical care. Med J Aust 2005;183:36972.
Address for correspondence: Dr Stephen J Mounsey, Dermatology Department, 250 Euston Road, London N1 2BU, UK.

Email: Stephen.mounsey@nhs.net
'This landmark report lays out in the starkest terms yet the devastating impact air pollution is having on our health, our economy and our society as a whole.'

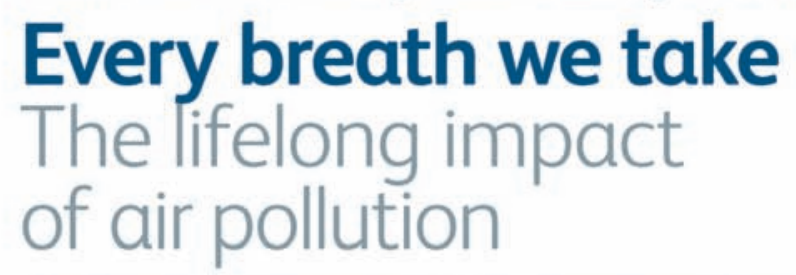

This major report plainly sets out the dangerous impact that air pollution has on our nation's health. Compiled by experts in medicine and environmental sciences, the report discusses the current evidence and draws up recommendations for action.

ISBN 978-1-86016-567-2 £15 including $p+p$ or free to download of Physicians
Download the report: rcplondon.ac.uk/pollution

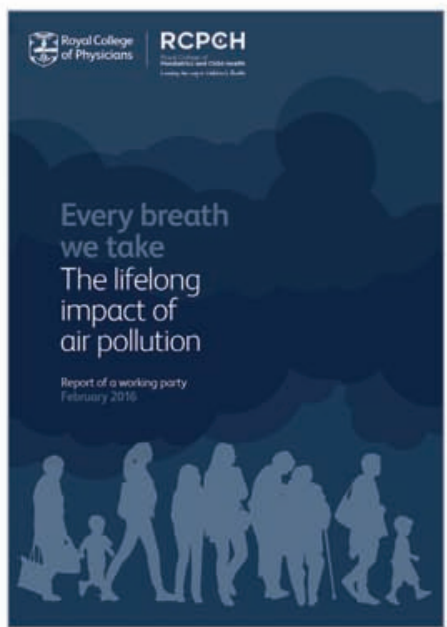

Order a copy: shop.rcplondon.ac.uk 